\title{
SmartClamp circumcision versus conventional dissection technique in terms of parental anxiety and outcomes: A prospective clinical study
}

\author{
Mert Ali Karadag, MD; ${ }^{*}$ Kursat Cecen, MD; ${ }^{*}$ Aslan Demir, MD; ${ }^{*}$ Yuksel Kivrak, MD; ${ }^{\dagger}$ Murat Bagcioglu, MD; \\ Ramazan Kocaaslan, MD;* Mustafa Ari, MD; Fatih Altunrende, MD*
}

"Kafkas University Faculty of Medicine, Department of Urology, Kars, Turkey; ${ }^{\dagger}$ Kafkas University Faculty of Medicine, Department of Psychiatry, Kars, Turkey; ${ }^{\S}$ Mustafa Kemal University Faculty of Medicine, Department of Psychiatry, Hatay, Turkey; ${ }^{*} B i l i m$ University Faculty of Medicine, Department of Urology, Istanbul, Turkey

Cite as: Can Urol Assoc J 2015;9(1-2):E10-3. http://dx.doi.org/10.5489/cuaj.2131

Published online January 12, 2015.

\section{Abstract}

Introduction: We prospectively analyzed parental anxiety and outcomes of the SmartClamp circumcision and the classic surgical dissection technique.

Methods: A total of 250 boys underwent circumcision between 2009 and 2012 at Kars State Hospital and Kafkas University Faculty of Medicine in Turkey. The initial 125 children were circumcised by conventional dissection method and the remaining children were operated on with a SmartClamp device. Children in both groups were compared in terms of bleeding, infection, penile edema, operative time, cosmetic result, length of the inner mucosal layer, and parental anxiety. We used a State-Trait Anxiety Inventory (STAI) form to gauge how the circumcision affected parental anxiety. This form was completed by parents on postoperative day 2 . Results: There were no statistically significant differences among the 2 groups in terms of age, bleeding, infection, and cosmetic displeasure $(p>0.05)$. The STAI scores of the parents from the SmartClamp group were statistically higher than that of the other group $(p<0.001)$. Penile edema was more common in the SmartClamp group $(p=0.039)$. However, the mean operative time was statistically shorter $(p<0.001)$ and the inner mucosal length was significantly longer in the SmartClamp group $(p<0.001)$.

Conclusion: Circumcision with the SmartClamp device was faster. Cosmetic results and complication rates were similar. Unfortunately, this technique seemed to entail the disadvantages of longer mucosal length, penile edema, and higher parental anxiety. Urologists should keep these points in mind when choosing a technique.

\section{Introduction}

Male circumcision is one of the most common and oldest surgical procedures in the world. ${ }^{1}$ This procedure involves the surgical removal of the preputium from the penis. The rate of male circumcision varies between $42 \%$ and $80 \%$.
The Middle East has the largest proportion of circumcised men..$^{2-5}$ Circumcision is performed for cultural, social, medical, and religious reasons. In Turkey, circumcision is most frequently performed during childhood for religious reasons and under local anesthesia either in hospitals, by urologists, general surgeons and pediatric surgeons, or as outpatient procedures by general practitioners.

There are various methods of circumcision, such as the classic surgical method, SmartClamp, and Mogen, Plastibell and Gomco clamps (preferred for newborn circumcisions). ${ }^{6-7}$ All of these procedures remove the preputium with excellent cosmetic results.

There is a gap in the literature, however, concerning parental distress and anxiety surrounding the circumcision of a young child. These worries are mostly due to different surgical approaches, and the expectations of cosmetic results and pain. In this study, we compared the parental anxiety and outcomes of the SmartClamp device and the classical surgical dissection technique. To the best of our knowledge, this is the first clinical study that comparing parental anxiety stemming from 2 different approaches to circumcision.

\section{Methods}

This study was approved by the local ethics committee of the Kafkas University Faculty of Medicine and performed in accordance with the Helsinki Declaration of the World Medical Association. All parents signed and understood the informed consent forms regarding the procedures and their inclusion into the study. A total of 250 boys aged 2 to 10 years underwent circumcision between 2009 and 2012 at the Kars State Hospital and Kafkas University Faculty of Medicine. After clinical and preoperative evaluation, the first 125 children were circumcised by the conventional dissection method (CDM) and the remaining children were operated by the SmartClamp method (SmartClamp Circumcision Device, Hengelo, Netherlands). Children with hypospadias, 
buried penis, ventral chordee or other genital anomalies and bleeding disorders were excluded from the study. All of the boys were preoperatively evaluated for any bleeding disorders and hepatitis.

Group 1 included 125 children with a mean age of $5.69 \pm 2.44$ years. These children were circumcised with CDM. Group 2 included another 125 children with a mean age of $5.58 \pm 2.83$ years; they were circumcised with the SmartClamp. All children in both groups were compared in terms of bleeding, infection, penile edema, operative time, cosmetic result, length of the inner mucosal layer, and parental anxiety. Cosmetic result and length of the inner mucosal layer were evaluated by a blinded urologist after 6 weeks. The main criterion in the evaluation of the cosmetic result was whether the glans penis was free or covered with unwanted skin. The independent urologist (MB) worked at the outpatient clinic of our department. The children were referred to him to check the cosmetic result and inner mucosal length. He did not know whether the child was circumcised with CDM or SC. We did not ask parents about cosmetic results because we wanted to get a standardized result from a blinded urologist. Bleeding was defined as cases requiring re-exploration and suturing for control of hemostasis. Infection was defined as cases having erythema with pus. After consultation with the psychiatry department, we decided to use the state-trait anxiety inventory (STAI) form to measure the impact of circumcision on parental anxiety. ${ }^{8-9}$ The children and parents were invited to the outpatient clinic on postoperative day 2 . After checking the child, parents were taken into a room with no accompanying staff so that they can complete the form together. Unfortunately, no standard consensus exists about the best application time to conduct this inventory for assessing the anxiety related to circumcision. Psychiatrists at our hospital mentioned that postoperative day 2 might be ideal. Postoperative days 5 or 7 may be too late to assess parental anxiety.

All of the procedures were performed under local anesthesia by subcutaneous injection of $0.2 \mathrm{~mL} / \mathrm{kg}$ lidocaine $1 \%$ with a small calibre needle at the $11 \mathrm{o}^{\prime}$ clock and $1 \mathrm{o}^{\prime}$ clock position at the base of the penis. Then the lateral sides and ventral surface of the penis were injected.

\section{Surgical techniques}

All of the circumcisions were performed by 1 urologist (KC). After local anesthesia and cleaning of the penis with the antiseptics, the adhesions between the inner mucosal layer and glans penis were removed by a sterile mosquito clamp.

\section{Conventional dissection technique}

The conventional sleeve technique was preferred. Inner mucosal layer was trimmed to $4 \mathrm{~mm}$ above the sulcus coro- norius. The hemostasis was maintained by using bipolar electrocautery and the edges of the skin were abducted with 4/0 absorbable sutures.

\section{SmartClamp}

The level of the circumcision was marked with a surgical pen preoperatively on the skin side of the preputium, just proximal to the sulcus coronorius. A measuring card with 8 circular holes was used to determine the correct clamp size that encircled the glans penis at the level of corona. The clamp sizes varied between 10 to $21 \mathrm{~mm}$. The foreskin needed to stretch widely to allow for the insertion of tube. Then, the outer clamp was inserted over the inner tube until it reached the end of the tube (Fig. 1). The skin was tightly gripped between the clamp and tube at this point. After pulling enough foreskin and observing the urethral meatus in the natural position, the clamp was locked. Unwanted skin was cut circumferentially 1 to $2 \mathrm{~mm}$ distal to the outer ring with the protection of the glans by the inner tube. ${ }^{6}$ The clamp was left in and the child was able to urinate through the open end. After 5 days, the connection between the clamp and the tube was cut and the SmartClamp was easily removed without pain.

\section{Statistical analysis}

This study was designed to detect up to a $30 \%$ difference in parental anxiety scores (STAI) between the 2 techniques with $90 \%$ power, assuming a significant difference level of 0.05 and a two-sided statistical test. Relying on the results of a pilot study performed in our department dealing with the parental anxiety of the SmartClamp, we calculated the sufficient sample size for our study. All these stages involved

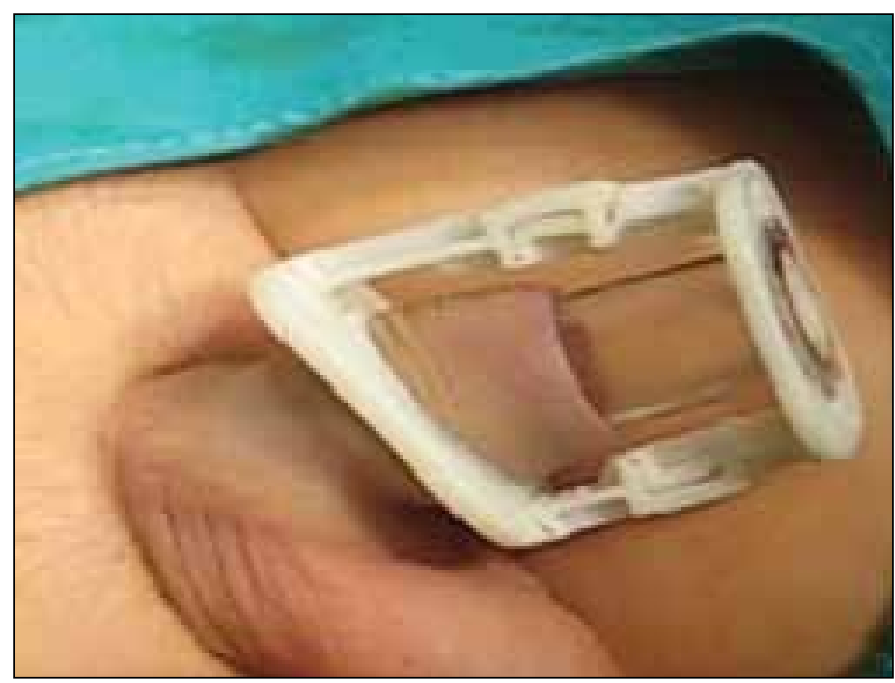

Fig. 1. SmartClamp device: Inner tube and outer clamp. Glans is secured inside the tube. 
Karadag et al.

\begin{tabular}{lccc}
\hline \multicolumn{1}{l}{ Table 1. The demographics and outcomes of the 2 groups } & & \\
\hline Parameters & CDT $(\mathbf{n}=125)$ & SC (n= 125) & $p$ value \\
\hline Age & $5.69 \pm 2.44$ & $5.58 \pm 2.83$ & $0.756^{*}$ \\
STAl score & $42.5 \pm 13.33$ & $51.98 \pm 12.71$ & $<0.001^{*}$ \\
Bleeding (\%) & $4(\mathrm{n}: 5)$ & $6(\mathrm{n}: 7)$ & $0.555^{* *}$ \\
Infection (\%) & $5(\mathrm{n}: 6)$ & $3(\mathrm{n}: 4)$ & $0.519^{* *}$ \\
Penile edema (\%) & $3(\mathrm{n}: 4)$ & $10(\mathrm{n}: 12)$ & $0.039^{* *}$ \\
Operation time(min.) & $18.08 \pm 3.55$ & $6.93 \pm 2.58$ & $<0.001^{*}$ \\
Cosmetic displeasure (\%) & $14(\mathrm{n}: 18)$ & $8(\mathrm{n}: 10)$ & $0.109^{* *}$ \\
Inner mucosal length (mm) & $5.09 \pm 1.22$ & $14.10 \pm 3.46$ & $<0.001^{*}$ \\
\hline
\end{tabular}

CDT: conventional dissection technique; SC: SmartClamp; STAI: State-Trait Anxiety Inventory; *Independent samples t-test; **Mann Whitney U-test.

consultations with a biostatistician. The data were analyzed by using SPSS for Windows version 16. Means, medians, and standard deviations were used for descriptive statistics. The characteristics of the two operative groups were compared. The characteristics with normal and non-normal distributions were compared by using independent samples student t-tests Mann Whitney tests, respectively. A $p$ value of $<0.05$ was considered significant.

\section{Results}

All parents agreed to complete the inventory form and participate in the study. The STAI scores of the parents of boys who underwent SmartClamp were nearly 10 points higher than that of other group; this result was statistically significant. The mean operation time was 11 minutes longer for the CDT group. Seven boys from the SC group required suturing after removal of the SmartClamp on postoperative day 5 . Five boys from the CDT group required re-suturing on the same day due to bleeding. In all cases, successful hemostasis was achieved. Boys with an infection were

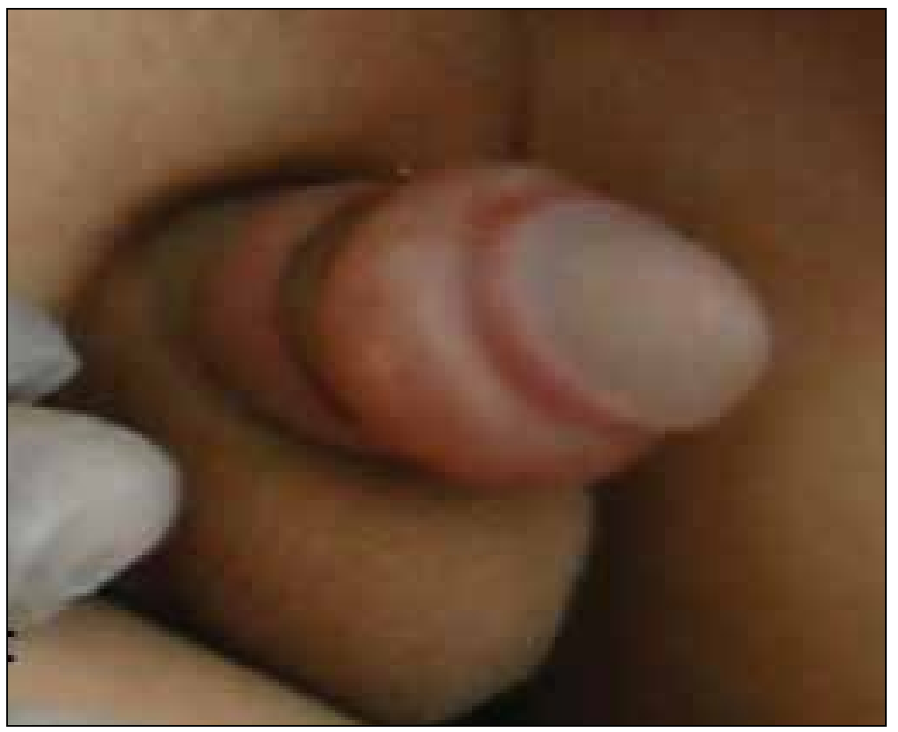

Fig. 2. Penile edema after the removal of the SmartClamp device. treated with oral antibiotics, and all recovered without complication (Table 1).

Penile edema was statistically significant in children of the SmartClamp group (Fig. 2, Table 1). Patients were treated with anti-inflammatory medicine (ibuprofene) and penile dressing. Except for 1 case, whose edema was resolved on postoperative day 17 , all patients recovered within 10 days postoperatively. Cosmetic displeasure was more common in children in the CDT group, but this result was not statistically significant. The mean inner mucosal length was nearly $10 \mathrm{~mm}$ longer in boys of the SmartClamp group. Parents of these children were informed of this outcome, but none of them wanted another intervention to remodel the inner mucosa because the cosmetic results were sufficient.

\section{Discussion}

We found that parental anxiety in the SmartClamp group was statistically higher. Penile edema was significantly more common in the SmartClamp group and the inner mucosal length was longer. The operative time of the SmartClamp group was statistically shorter than for the CDT group. No significant differences were encountered in terms of complications, like bleeding, infection, and cosmetic displeasure - all of which were evaluated by a urologist who was blinded to the study.

Our study's strengths lie in its prospective design, sample size, and emphasis on parental distress ensuing from the 2 different approaches of circumcision. The evaluation of cosmetic results and inner mucosal length by a blinded urologist also added power to the study. The circumcisions were all performed by a single urologist; this removed the surgical ability factor of different urologists which could have determined cosmetic result, mucosal length, operation time, and complications.

There are also several limitations of our study. The study's design was not prospectively randomized and the quality of life of the children was not investigated. Body mass index could have affected the cosmetic result and the outcomes were not evaluated. If different parameters, such as cost analysis of the procedures, usage of analgesics, return to nor- 
mal daily life, and pain while voiding, were included in this study, the power of the paper would have been enhanced.

Circumcisions are mostly performed due to religious reasons in our country and they are among the most important issues for parents. Parental anxiety in the SmartClamp group was statistically higher in our study. In our opinion, this difference was due to the SmartClamp device causing extra anxiety in parents. Visualization of the SmartClamp device around the penis may have made the parents think that their child would feel pain and discomfort. Moreover, the dressing of the penis was removed on postoperative day 1 in the CDT group and, unlike the SC group, the parents easily observed the state of the penis. Another stressful event for families included visits by relatives or neighbours who came to see the newly circumcised boys, a very common social phenomenon in Turkey. If a boy is carrying a device around his penis, the visitors may criticize it or wonder about the results of this approach, thus increasing parental anxiety. Expectations of the cosmetic result also affected anxiety levels.

Bleeding episodes also caused parental distress and anxiety. Bleeding was the most common complication, ranging from 0.08 to $0.18 .^{1,10,11}$ This rate could change according to the preferred approaches, including the classic surgical method, Gomco clamp, Mogen clamp or Plastibell device. ${ }^{12-14}$ The bleeding rate of the CDT and SmartClamp boys in our study was $4 \%$ and $6 \%$, respectively. Aldemir and colleagues investigated and compared the outcomes of the SCD with CDT. ${ }^{6}$ The bleeding rate with the SCD was $2 \%$ in their study. Our bleeding rate $(6 \%)$ seemed higher and this could explain the experience of the other group. Infection rates in the two studies were similar. In regards to cosmetic results, Aldemir and colleagues achieved a 88\% rate of free glans penis with SCD; this value was $92 \%$ in our study. They did not mention penile edema in their paper, however. In our opinion, this was the most troubling complication of SCD in the postoperative period. The outcomes of CDT in the 2 studies were similar. Our study's mean length of inner mucosal layer was $9 \mathrm{~mm}$ longer than the Aldemir study's outcome. The disparity between the 2 studies might be explained by a difference in the surgical skills of the urologists. The authors concluded that circumcision with SCD was efficient and safe and could change the classical approach to circumcision.

\section{Conclusion}

Circumcision with SC device was faster, when compared to CDT. Cosmetic results and complication rates were similar. Unfortunately, this technique seemed to carry the disadvantages of longer mucosal length, penile edema, and higher parental anxiety. These points should be kept in mind by the urologists before choosing this technique.

Competing interests: Authors declare no competing financial or personal interests.

This paper has been peer-reviewed.

\section{References}

1. AAP Task Force on Circumcision. Male circumcision. Pediatrics 2012;130:756-85. http://dx.doi. org/10.1542/peds.2012-1990

2. Centers for Disease Control and Prevention (CDC). Trends in-hospital newborn male circumcision-United States, 1999-2010. MMWR Morb Mortal Wkly Rep 2011;60:1167-8.

3. Nelson $(P$, Dunn $R$, Wan J, et al. The increasing incidence of newborn circumcision: Data from the nationwide inpatient sample. J Urol 2005;173:978-81. http://dx.doi.org/10.1097/01. ju.0000145758.80937.7d

4. Xu F, Markowitz LE, Sternberg MR, et al. Prevalence of circumcision and herpes simplex virus type 2 infection in men in the United States: The National Health and Nutrition Examination Survey (NHANES), 1999-2004. Sex Trans Dis 2007;34:479-84.

5. Burgu B, Aydogdu O, Tangal $S$, et al. Circumcision: Pros and cons. Indian I Urol 2010;26:12-5.

6. Aldemir $M$, Cakan $M$, Burgu $B$. Circumcision with a new disposable clamp: Is it really easier and more reliable? Int Urol Nephrol 2008;40:377-81. http://dx.doi.org/10.1007/s1 1255-007-9275-x

7. Freeman JJ, Spencer AU, Drongowski RA, et al. Newborn circumcision outcomes: Are parents satisfied with the results? Pediatr Surg Int 2014;30:333-8. http://dx.doi.org/10.1007/s00383-013-3430-5

8. Spielberger CD, Gorsuch RL, Lushene RE. Manual for Stait-Trait Anxiety Inventory, Palo Alto, California: Consulting Psychologist Press; 1970.

9. Oner N, Le Compete A. Durumluk Sürekli Kaygı Envanteri El Kitabı, stanbul: Bo aziç, Üniversitesi Yayılarl; 1985.

10. Weiss $\mathrm{HA}$, Larke $\mathrm{N}$, Halperin $\mathrm{D}$, et al. Complications of circumcision in male neonates, infants and children: A systematic review. BMC Urol 2010;10:2. http://dx.doi.org/10.1186/1471-2490-10-2

11. Wu X, Wang Y, Zheng J, et al. A report of 918 cases of circumcision with the shang ring: Comparison between children and adults. Urology 2013;81:1058-63. http://dx.doi.org/10.1016/j.urology.2012.11.046

12. Plank RM, Ndubuka NO, Wirth KE, et al. A randomized trial of mogen clamp versus plastibell for neonatal male circumcision in Botswana. J Acquir Immune Defic Syndr 2013;62:131-7. http://dx.doi. org/10.1097/QAA.0b013e318285d449

13. Bhat NA, Hamid R, Rashid KA. Bloodless, sutureless circumcision. Afr J Paediatr Surg 2013;10:252-4.

14. Xi RC, Sheng YR, Chen WH, et al. Male circumcision performed with 8-figure non-absorbable suture technique. Can Urol Assoc J 2014;8:e142-7. http://dx.doi.org/10.5489/cuaj.1521

Correspondence: Dr. Mert Ali Karadag, Kafkas Üniversitesi Tıp Fakültesi Hastanesi, Üroloji A.B.D, Kars, Türkiye; karadagmert@yahoo.com 